\title{
Research on the Overall Design of Navigation Computer Hardware Module Based on DSP and FPGA
}

\author{
Fen Ren ${ }^{1, a}$ and Wen Sun ${ }^{2, b^{*}}$ \\ ${ }^{1}$ Xianning Vocational Technical College \\ ${ }^{2}$ Tellhow Polytron Technologies Inc \\ as7994@163.com, ${ }^{\mathrm{b}}$ zhaoleies@163.com \\ *The Corresponding author
}

Keywords: Navigation computer; DSP; FPGA; Missile guidance system

\begin{abstract}
The navigation computer plays an important role in the missile guidance system. With the development of missile guidance systems, the navigation computer is required to have higher practical performance. However, DSP navigation computer hardware module couldn't meet the requirements any more .Due to the above reasons, this paper puts forward the suggestion that using FPGA module to improve the disadvantages of DSP modules. The FPGA module is used to increase the data cross port with the ground and boot device, so that the DSP module can adapt to the complex computing environment. In this paper, a navigation computer hardware module is designed for missile guidance system, while the design idea of hardware module are described in detail, as well as the design of each part of the main points. Which is helpful for the applications of DSP and FPGA combined with navigation computer.
\end{abstract}

\section{Introduction}

The reason why the missile has a high hit rate is due to the role of the navigation system. The navigation system is composed of three parts: the measurement of the missile state and the calculation of the theoretical trajectory as well as the correction of the target position of the missile[1]. The integrated navigation system can improve the precision of navigation, and further improve the accuracy of the missile hit rate and effective range requirements. During the flight, the missile system will generate a large amount of dates[2]. So, we are faced with the fact that how fast the navigation computer analyze data from the computer guidance system and the ground control center data processing, timely monitoring well trajectory, and dynamically adjust the missiles. There is an urge need to deal with technical problems.

DSP technology has been more mature and widely used through decades of development[4]. Because of obvious advantages in the missile guidance system that DSP has a high precision in complex operation and fast operation, it has been widely used in navigation computer. Although the development of DSP has been more mature, the external interface is also more and more, but the specific to the pin, most of the external interface is used in the multiplexing method. If one of the external interfaces is occupied, other types of external connections must not be used, so that when the external interface is communicated with the components on a variety of missiles, $\mathrm{t}$ he external interface is not sufficient. As is known to us, FPGA is the field programmable gate array. Making full use of FPGA programmable, designers can flexibly configure the hardware functionality needed to get rid of the need for a dedicated chip according to the specific requirements. The combination of FPGA and DSP, and FPGA as the external interface of the expansion device, can make up for the weak control capability of DSP.

According to the above situations, the navigation computer hardware module used in the missile guidance system of DSP and FPGA is designed in this paper, which makes full use of DSP's powerful ability to analyze and process date; besides, FPGA hardware programmable advantages are also taken of. It systematically improves the navigation computer data analysis and calculation capabilities on the missile, also, enriches the computer's external interface[5]. Therefore, it meets the requirements that the guidance system need the computer have high precision, small size, and 
fast computing capabilities. Such research makes a positive difference in the further development of navigation computer.

\section{The Conceptual Design of Navigation Computer}

Playing a controlling role, navigation computer is equivalent to the brain of the navigation system. A complete and effective navigation computer needs to have both the ability to acquire a large number of measurements for rapid analysis processing, as well as access to measurement data and quick analysis processing. In view of the functional characteristics of the navigation computer, we consider designing the paper from the following aspects[4].

(1) Data acquisition of three direction acceleration and battery voltage

(2) The information system interacts with the ground base station, including the transmission of the missile through the long line 422 and the post-launch missile communication device to the ground base station.

(3) The information exchange between the missile and the satellite equipment and the inertial navigation equipment.

(4) The measured mass data are analyzed and processed.

\section{The Overall Design of the Navigation Computer}

According to the analysis of the navigation computer function in the part one, the navigation computer needs to measure all kinds of information, which includes accelerometer transmission data, the battery voltage signal, the interactive communication with the ground equipment, the satellite equipment, the inertial navigation equipment, the remote control equipment and the lead head device, and Signal analysis and processing modules and other design modules

Therefore, the navigation computer hardware requires two measurement components, namely five interactive communication components, as well as rapid analysis and processing of data components. The use of DSP in the rapid analysis of data processing and the advantages of the design of the DSP module to undertake data processing tasks. However, due to the limitations of DSP external interface, navigation computer needs a larger number of external, and five interactive communication requires real-time information transmission. So the design of the FPGA as an additional component is to undertake the task of interactive communication, and interactive information real-time input DSP module for analysis and processing. The measurement signal of the accelerometer and the supply voltage in the guidance system is directly input to the DSP module through the amplifier and A / D conversion. Concerning analysis and design of the main modules of navigation computer, the circuit structure of the navigation computer hardware module is shown as follows Fig. 1.

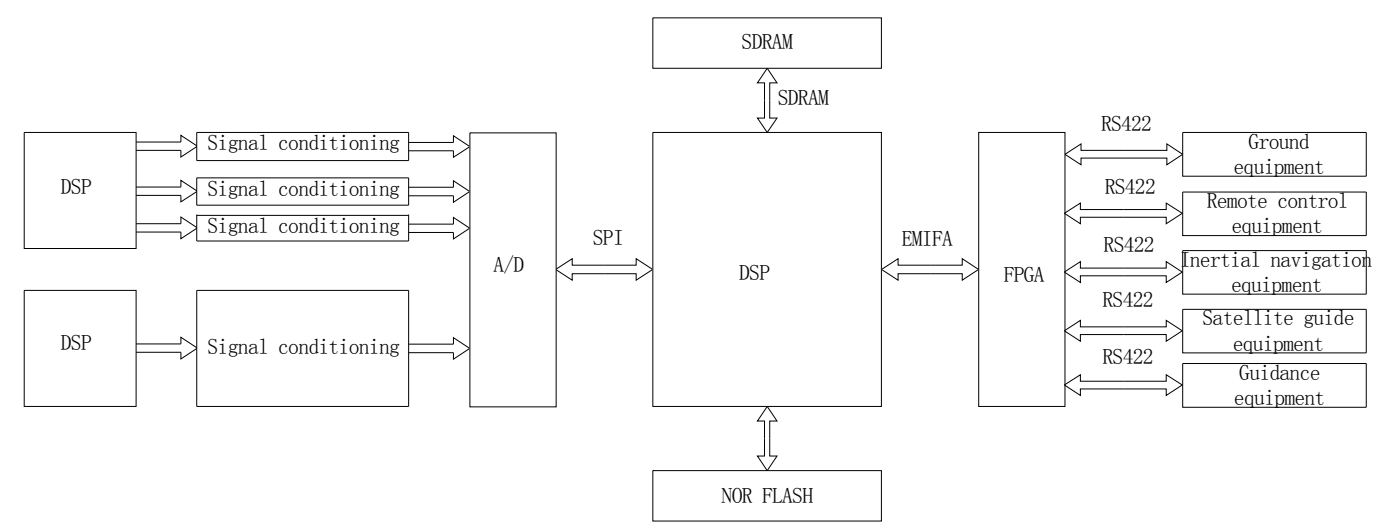

Figure 1. Navigation computer hardware circuit diagram

From Fig. 1, the interactive communication device communicates with the FPGA using the 422 device, EMIFA interface between FPGA and DSP. As the DSP storage space is limited, lacking of memory is common in the analysis of massive data, so we design external expansion of the 
SDRAM to storage data. Design of NOR FLASH program memory can quickly read the data, improve the speed of operation, in addition to FPGA with time-sharing multiplexing EMIFA interface.

\section{Optimization Design of Navigation Computer}

The components of the navigation computer interact with each other, Only by coordinating the performance of each component can it achieve the optimal state. Therefore, it is of great significance to select the most suitable components.

Optimal DSP selection. Considering the features of navigation computer, it is necessary to meet the requirements of high speed, high precision, low power consumption, rich interface and small size.

Currently, three companies, namely TI, ADI, Motorola, mainly produce DSP. The biggest is TI, which has the highest market share. The TMS320C6747 32 bit floating point processor produced by TI company has obvious advantages. 6747 processor has the characteristics of high speed, high precision and low power consumption, these advantages meet the requirements of DSP. The parameters are now shown in Table 1 below.

Table1 Comparison of 6713B and 6747 parameters

\begin{tabular}{|c|c|c|}
\hline Model & 6747 & $6713 \mathrm{~B}$ \\
\hline Maximum frequency & $456[\mathrm{MHz}]$ & $300[\mathrm{MHz}]$ \\
\hline $\begin{array}{c}\text { Core voltage } \\
\text { (frequency 300[Hz]) }\end{array}$ & $1.2[\mathrm{~V}]$ & $1.4[\mathrm{~V}]$ \\
\hline First level cache & $64[\mathrm{KBtey}]$ & $8[\mathrm{KBtey}]$ \\
\hline On-chip RAM & $256[\mathrm{Kbyte}]+128[\mathrm{KByte}]$ & $256[\mathrm{Kbyte}]$ \\
\hline EMIF & 2 & 1 \\
\hline size & $17[\mathrm{~mm}] \times 17[\mathrm{~mm}]$ & $17[\mathrm{~mm}] \mathrm{x} 17[\mathrm{~mm}]$ \\
\hline
\end{tabular}

As shown in Table 1 above, 6747 are better than 6713B in the main frequency, energy consumption, RAM, size and other aspects. Therefore, the choice of TI's 6747 processor is more reasonable.

Optimal FPGA selection. As an extension of the serial port, FPGA don't have high requirements for capacity of the logical resource requirements. XC3S200 equivalent logic gate 50K, there are two types of distributed and block RAM, with a clock manager and the four multiplier. Because of the number of working structure and high work rate, $\mathrm{I} / \mathrm{O}$ can meet the needs of multiple serial ports. Chip size is only $16 \mathrm{mmX} 16 \mathrm{~mm}$. The voltage is $1.2 \mathrm{~V}$. Therefore, the selection of XC3S200 is able to meet the design requirements about the parameters .

Design of power supply. Power supply is an important part of navigation computer. If there is no power to navigate the computer, the other components will not function properly. Taking into account the missile in the flight process, the energy required to guide the system is supplied by itself. Therefore, we should try to reduce the energy consumption of navigation computer. After considering the advantages and disadvantages of switching power supply and linear power supply, switching power supply is used for power supply chip. According to the requirements of the input voltage and the energy consumption of each component, the TPS54331 switching buck power supply produced by TI company meets the requirements. The input voltage of TPS54331 is $3.5 \mathrm{~V} 28 \mathrm{~V}$, while the output voltage is $0.8 \mathrm{~V}$. And the output current is up to $3 \mathrm{~A}$, also, the conversion rate can reach $90 \%$.

Based on the above analysis, the power supply is designed using 3 voltage values of $1.2 \mathrm{~V}$, $2.5 \mathrm{~V}$ and 3.3V TPS54331 models of power supply. The power supply diagram is shown in Fig. 2 below. 


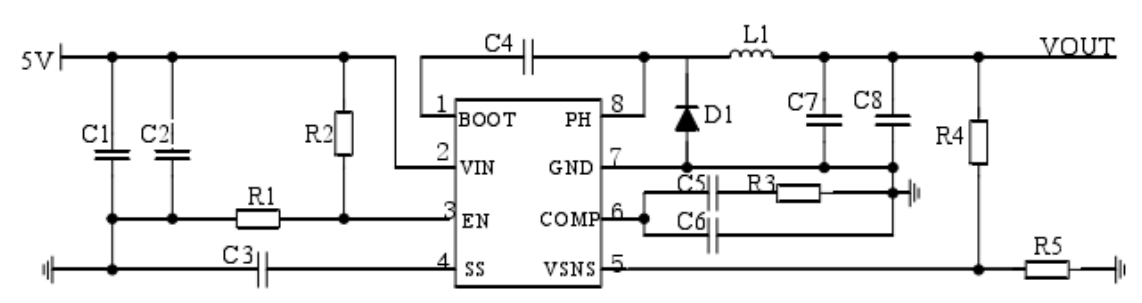

Figure 2. Power circuit design

As can be seen from Fig. 2 above, R3and R4 determine the accuracy of the output voltage, and $V_{\text {ref }}=0.8 \mathrm{~V}$ 。 The output voltage of the power supply is as shown in the following Eq.1.

$$
V_{\text {out }}=V_{\text {ref }} \times\left(\frac{R 4}{R 5}+1\right)
$$

The Delay time difference $T_{s s}$ between the output voltage and the input voltage is determined by the capacitance of the SS terminal. The formula for calculating $T_{s s}$ is as follows Eq.2.

$$
T_{s s}(m s)=\frac{C_{3}(n F) \times V_{r e f}(V)}{I_{s s}(\mu A)}
$$

Among:

$$
\begin{aligned}
& V_{r e f}=0.8 \mathrm{~V} \\
& I_{s s}=2 \mu \mathrm{A}
\end{aligned}
$$

\section{Summary}

Navigation computer plays an important role in missile guidance system. How to meet the requirements of the current guidance system to run fast, high precision, low energy consumption, serial connection, small size and so on, to achieve navigation computer hardware module optimization. This is important for breaking the navigation computer technology problems.

In this paper, according to the characteristics of DSP external interface and high running speed, combining with FPGA as an extension component to solve the shortcomings of DSP, so as to optimize the function of navigation computer. At the same time, this paper makes a general design study on the hardware module of the navigation computer, and puts forward how to choose the appropriate equipment model, and make the circuit design and analysis of the computer power supply, which is instructive to improve the performance of navigation computer.

\section{References}

[1] Min.Liu, Jizhou.Lai and Zhimin.Li. An adaptive cubature Kalman filter algorithm for inertial and land-based navigation system[J]. Aerospace Science and Technology. Vol. 52 (2016), p60.

[2] WANG.Yue-ai1and WANG Bo. Power Technology Research And Development Trends[J]. China lntegrated Circult. Vol. 4(2012), p70.

[3] Gu.Song. Interface design between TMS320C67 series EMIF and asynchronous FIFO memory[J]. ELECTRONIC ENGINEER. Vol. 31 (2004) No5, p53.

[4] YANG.Long, LI.Fan-ming and LIU.Shi.jian. Realization of high-speed communication between DSP and FPGA by EMIF dual-port RAM[J]. Modern Electronics Technique. Vol 27 (2014), No13, p10-11.

[5] WANG.Dawei, REN.Yongfeng and SHAN.Yanhu. Design of Over-Loading Signal Adjustment Circuit[J]. Chinese Journal of Electron Devices. Vol39 (2016), No2, p357. 